\title{
Establishing Running
}

\section{Intensities of Elite Field Hockey Players During Competitive Match-Play}

Arain Forouhaneh

\section{ABSTRACT}

Field hockey requires players to perform at varying intensities throughout a competitive match, involving high-speed running combined with tactical skills in order to outscore the opposition. A greater understanding of specific running demands imposed by competitive matchplay may aid coaches in appropriate prescription of training and adequate recovery programmes. Purpose: to determine peak duration- and positionspecific running intensities during field hockey competition, using a rolling average method. Methods: twenty-one elite male field hockey players were analysed through $15 \mathrm{~Hz}$ Global Positioning System (GPS) technology across a 16 match competitive season. Peak values for relative distance $(\mathrm{m} \cdot \mathrm{min}-1)$ and high-speed distance (m.min-1) were calculated, placed in a velocity-time curve and analysed using a rolling average method across ten different durations $(1,2,3,4,5,6,7,8,9$, $10 \mathrm{~min}$ ) for each playing position. Results: Forwards and midfielders covered significantly $(p<0.05)$ more relative distance than defenders for averages 1 to 6 , with the forwards covering the greatest maximum relative distance and high-speed relative distance. There was a substantial decrease in relative distance and highspeed distance as the length of the rolling average increased, presenting small to moderate differences between durations 4 to $10 \mathrm{~min}$, with the magnitude of differences between lengths decreasing as the rolling average length increased. Conclusion: These findings suggest that match-play running demands are significantly more intense than previously reported for all positions. As forwards exhibited a greater running intensity throughout, position specific training drills should replicate the most demanding phases of field hockey competition.

\section{INTRODUCTION}

Field Hockey is a high-intensity team sport played at various standards, ranging from amateur all the way to elite level. Competitive match-play in the English Hockey Leagues (EHL) consists of two 35minute halves contested by two teams each fielding ten outfield players and one goalkeeper. The schedule throughout an $\mathrm{EHL}$ season consists of one match per week, 16 matches in a season, resulting in sufficient recovery time before the next match with an increased importance in 
match outcomes as the season progresses. Field Hockey requires players to perform at varying intensities throughout a competitive match, involving high-speed running and changes in direction combined with the tactical skills in condensed areas of a pitch in order to outscore the opposition. ${ }^{1}$ During a field hockey match, depending on position played, players typically cover an average distance of $5540 \mathrm{~m}$, which equalizes to an average relative distance of $126 \mathrm{~m} \cdot \mathrm{min}^{-1} .^{5}$ Despite the increasing popularity of the physical ${ }^{2}$, 3,5 and running demands $1,4,5$ of field hockey, there is a lack of research focussing on the most intense periods of play in competitive match-play.

The importance of Global Positioning Systems (GPS) technology for sport scientists and coaches has been thoroughly documented in team sport competition ${ }^{11,12}$. It is commonly used to assess high-speed movements during training and competition based activity, with many studies summarising the importance of correlation between highspeed running and high-intensity exercise to improve performance in team sports ${ }^{7}$, 10. The use of GPS technology has become increasingly popular in providing quantitative analysis on the movement demands of match-play within field hockey 1-7, allowing for accurate measurement of speed and distance during activity. Data collected through GPS technology provides coaches with the information to construct training drills to best replicate these demands.

A greater understanding of the specific running demands imposed by competitive match-play will result in appropriate prescription of training and adequate recovery programmes utilized by coaches in order to enhance and maximise performance. ${ }^{6}$ In recent years, timemotion analysis has provided valuable information, establishing running intensities within various different team sports such as field hockey ${ }^{1-7} 13,15$, soccer 9,14 and rugby ${ }^{8,17}$. Coaches have relied on data which describes absolute running demands to monitor their athletes during competition. Whilst these methods are sufficient to quantify player movements, entire match values substantially underestimate the running requirements of the most intense periods of matchplay..$^{8,9}$ As transient fatigue is experienced throughout team sport matches, this suggests decline in running performance which might be an indicator of the most intense periods of play. ${ }^{19,20,21}$ Bradley et al., (2010) reported elite soccer players high-speed running after the most intense 5-min period of the match to significantly drop to values below the overall game mean, identifying a decline in 53\%. In addition to this, elite players covered $12 \%$ more high-speed running distance in the first 15-min period of the game than the final 15-min periods of both halves. This shows that high-speed running decreases after the most intense period of a game, with the intensity of the game fluctuating throughout indicating temporary forms of fatigue. A similar trend was observed by Mohr et al., (2003) in top-class soccer players. The peak relative distance $\left(\mathrm{m} \cdot \mathrm{min}^{-}\right.$ $\left.{ }^{1}\right)$ covered in high-speed running (219 \pm 8 $\mathrm{m} \cdot \mathrm{min}^{-1}$ ) was significantly greater than the average distance covered during all 5-min intervals $\left(121 \pm 4 \mathrm{~m} \cdot \mathrm{min}^{-1}\right)$. The variation in running demands observed through research in team sport competition suggests that peak match-play intensity is not adequately captured through absolute match demand analysis. ${ }^{8}$ 
Given the previously proposed relationships between transient fatigue and running performance, such observations are made through predefined periods of play (e.g. 0-5 min, 0-15 min etc.) which may result in underestimating the intensity of that period. Varley et al., (2012) was the first to investigate match performance in soccer across selected intervals of play using a rolling average analysis method. Results show a substantial underestimation of peak distance through predefined periods $\left(1^{\text {st }}\right.$ Half: $142 \pm 24 \mathrm{~m} \cdot \mathrm{min}^{-1}, 2^{\text {nd }}$ Half: $138 \pm 41$ $\left.\mathrm{m} \cdot \mathrm{min}^{-1}\right)$ in comparison to rolling average ( $1^{\text {st }}$ Half: $177 \pm 91 \mathrm{~m} \cdot \mathrm{min}^{-1}, 2^{\text {nd }}$ Half: $166 \pm$ $43 \mathrm{~m} \cdot \mathrm{min}^{-1}$ ) by $20-25 \%$. Since, the rolling average has been more commonly used to identify the most intense periods of play. Delaney et al., (2015) reported a peak average of $179 \pm 15 \mathrm{~m} \cdot \mathrm{min}^{-1}$ covered by rugby players, which is substantially greater than the numbers previously reported $\left(80-100 \mathrm{~m} \cdot \mathrm{min}^{-1}\right)^{24}$ in rugby league.

Understanding the running demands of field hockey players according to positional role is necessary in the development of training protocols due to the variations in tactical requirements of specific positions and playing styles. ${ }^{1}$ There have been consistent findings regarding the amount of high-intensity distance covered varied by playing position in team sports, especially field hockey. Typically, differences in total distance and highspeed running distance observed are mainly due to positional differences. Macutkiewicz and Sunderland (2011) reported that forwards spend a greater time engaging in high-speed running (8\%) than midfielders (6\%) and defenders (5\%). Similarly, Spencer et al., (2004) reported that forwards performed a higher number of sprints than other positions. It has been suggested than differences in playing time can affect high-speed distances, which are influenced by the running demands of each position. ${ }^{4}$ Consistent findings that physical and technical outputs decrease towards the end of field hockey matches, suggests that the change in substitution rule with a roll-on roll-off nature, will reduce physical fatigue and lead to an increase in running performance. ${ }^{25}$ This means that players will spend on average 48-min of the 70-min on the pitch, with forwards spending the least amount of time on the pitch due to greater engagement with high-intensity exercise (41-min). ${ }^{3}$ This data may be used by coaches to make substitutions based on these variables. ${ }^{24}$

As previously stated, the absolute running demands of different positions in field hockey have been examined in recent years for the prescription of training drills. Alternatively, a more practical method to ensure field hockey players are meeting competitive match-play intensities during training would be to develop position- and duration-specific movement indicators to establish specific running demands required in competition. To quantify peak running intensities of competitive matchplay, the use of a rolling average method allows researchers to identify the most intense period of competition for various moving average lengths. Such data will assist coaches in providing intensity specific training drills relative to peak match activity profiles. Therefore, the purpose of this study is to determine peak duration- and position-specific running intensities during field hockey competition, using a rolling average method.

\section{METHODS}




\section{Design}

The current observational GPS analysis study examined the duration-specific and position-specific velocity based movements to determine relative distance (RD) and high-speed running (HSR) from a $15 \mathrm{~Hz}$ velocity-time curve. Data was collected across the 2018/2019 England Field Hockey League Conference North (EHL) competitive season. All subjects were informed of the aims and requirements of the research and informed consent was obtained. The Nottingham Trent University Ethics Committee approved of all procedures.

\section{Subjects}

Data was collected from twenty one elite male field hockey players (age $=22.0 \pm 3.2$ yrs, body mass $=75.7 \pm 4.4 \mathrm{~kg}$, stature $=$ $1.83 \pm 0.06 \mathrm{~m}$ ) from the same club during 16 matches throughout the 2018/2019 England Field Hockey League Conference North (EHL) competitive season (8 wins, 4 draws, 4 losses, Final Position: $4^{\text {th }}$ ). Between matches, players typically trained 2-3 field sessions and 1-2 resistance-based sessions. Each match was 70-min in duration, separated into two 35-minute halves. Players were categorised by playing position; either defender $(n=5)$, midfielder $(n=10)$ or forward $(n=6)$. The mean number of observations for each player was $11.19 \pm 5.02$.

\section{Methodology}

The running demands of a player were recorded using a portable GPS unit with a sampling rate of $15 \mathrm{~Hz}$ (SPI HPU, GPSports,
Canberra, Australia). The portable $15 \mathrm{~Hz}$ GPS units were worn in a customised padded pouch within a catapult vest, positioned in the centre of the upper back area, slightly superior to the scapulae. To minimise the effect of inter-unit reliability, each player was allocated the same unit for the duration of the season. The validity and reliability of $15 \mathrm{~Hz}$ GPS have been previously established (Johnston RJ, Watsford ML, Kelly SJ, Pine MJ, Spurrs RW, 2013).

Upon completion of each match, GPS data were extracted and analysed using the appropriate proprietary software (Team AMS, Canberra, Australia). A total of 240 individual files were collected, with each file being trimmed to only include time spent on the pitch throughout the 70-min match (excluding substitution periods, half time and extra time periods). The average time spent on the pitch per game for each position was; $57.08 \pm 6.16 \mathrm{~min}$ for defenders, $43.17 \pm 6.30 \mathrm{~min}$ for midfielders, and $41.14 \pm 6.16 \mathrm{~min}$ for forwards. Velocity-time curves were linearly interpolated to $15 \mathrm{~Hz}$, and a fourthorder Butterworth filter applied with a $1 \mathrm{~Hz}$ cut-off frequency. These files were then further processed through Microsoft Excel, to allow the computation of a moving average for each player's relative variables considered in the present study $\left(\mathrm{km} \cdot \mathrm{h}^{-1}\right)$. The variables computed for each player included, relative distance $\left(\mathrm{km} \cdot \mathrm{h}^{-1}\right)$ and high-speed distance $\left(\mathrm{km} \cdot \mathrm{h}^{-1}\right)$. For the computation of a moving average over each output variable using ten different durations $(1,2,3,4,5,6,7,8,9,10 \mathrm{~min})$ for all players, with the maximum value for each duration recorded. For example, for a 1 min rolling average, 900 consecutive data points were identified (i.e. 15 samples per second for 60 seconds) where the subject 
exhibited the greatest values. For a $2 \mathrm{~min}$ rolling average, 1800 consecutive data points were used, etc. Data was categorised by playing position and averaged across all observations for that positional group, for between-group comparisons.

\section{Statistical Analysis}

A repeated measures ANOVA was used to calculate and determine differences between maximum relative differences calculated from all maximum moving average durations. A Bonferroni post-hoc test was used to locate significant differences between playing positions for all moving average durations. Data distribution was assessed for normality using the Shapiro-Wilk test. Cohen's effect sizes (ES) were used to describe differences in running intensities between moving average durations. In association with previous literature (Hopkins WG, Marshall SW, Batterham AM, Hanin J, 2009), effect sizes of $>1.20,0.61-1.19$, 0.20-0.60 were considered large, moderate and small, respectively. Descriptive statistics are presented as mean $\pm S D$, with certain data reported as mean $\pm 90 \%$ confidence limits $(\mathrm{CL})$ where stated. Statistical analysis was performed using SPSS (v.16; SPSS Inc., Chicago, IL).

\section{RESULTS}

Maximal relative distances covered $\left(\mathrm{m} \cdot \mathrm{min}^{-1}\right)$ for each position can be seen in Table 1. It illustrates a substantial decrease in maximal relative distance covered as the length of the rolling average used increases for all playing positions. Quantitative analysis revealed that midfielders and forwards covered significantly $(p<0.05)$ more relative distance than defenders for averages 1 to $6 \mathrm{~min}$, but the magnitude of these differences decreased as the length of the rolling averages increased. Forwards covered a greater relative distance than Midfielders for durations 1 to $4 \mathrm{~min}$ and 6 to $7 \mathrm{~min}$, however there was no significant difference for all rolling average lengths.

\begin{tabular}{|c|c|c|c|c|}
\hline $\begin{array}{l}\text { Rolling } \\
\text { Length }\end{array}$ & Average & Defenders & Midfielders & Forwards \\
\hline 1 & & $173 \pm 24^{b c}$ & $182 \pm 21^{a}$ & $188 \pm 25^{a}$ \\
\hline 2 & & $156 \pm 23^{b c}$ & $167 \pm 20^{a}$ & $171 \pm 22^{a}$ \\
\hline 3 & & $147 \pm 20 \mathrm{bc}$ & $159 \pm 20^{a}$ & $159 \pm 21^{a}$ \\
\hline 4 & & $142 \pm 19 b c$ & $153 \pm 20^{a}$ & $154 \pm 19^{a}$ \\
\hline 5 & & $138 \pm 18^{b c}$ & $149 \pm 19^{a}$ & $149 \pm 18^{a}$ \\
\hline 6 & & $136 \pm 17^{b}$ & $145 \pm 18^{a}$ & $146 \pm 17$ \\
\hline 7 & & $134 \pm 17$ & $142 \pm 17$ & $143 \pm 17$ \\
\hline 8 & & $133 \pm 15^{b}$ & $139 \pm 17^{b}$ & $140 \pm 17$ \\
\hline 9 & & $131 \pm 14$ & $138 \pm 17$ & $139 \pm 18$ \\
\hline 10 & & $130 \pm 13$ & $139 \pm 16$ & $134 \pm 20$ \\
\hline
\end{tabular}

Table 1. Maximum relative distances $(\mathrm{m} \cdot \mathrm{min}-1)$ of field hockey players by position for each moving average $( \pm$ SD).

$a=$ significantly different from defenders; $b$ = significantly different from midfielders; $c$ = significantly different from forwards. Significance set at $p<0.05$. 
Figure 1 shows high-speed relative distance $(\mathrm{m} \cdot \mathrm{min}-1)$ for each position across 10 different moving average lengths. Forwards covered significantly $(p<0.05)$ greater high-speed relative distance than midfielders and defenders for average lengths 1 and $2 \mathrm{~min}$. There was no significant $(p<0.05)$ difference in highspeed relative distance between positions for rolling average lengths 3 to $10 \mathrm{~min}$. Forwards covered a greater high-speed relative distance than midfielders for all rolling average lengths, with midfielders greater than defenders for all lengths showing no significant $(p<0.05)$ differences throughout.
Figure 1 and figure 2 illustrate a substantial decrease in relative distance and highspeed relative distance as the length of the rolling average used increases. Quantitative analysis revealed significant differences $(p<0.05)$ between all rolling average durations. Table 2 illustrates all pairwise comparisons and the magnitude of differences between all 10 rolling average durations for relative distance covered. Analysis revealed that there were small to moderate differences $(p<0.05)$ between durations 4 to $10 \mathrm{~min}$, with the magnitude of the differences between lengths decreased as the length of the rolling averages increased for both relative distance and high-speed distance covered.

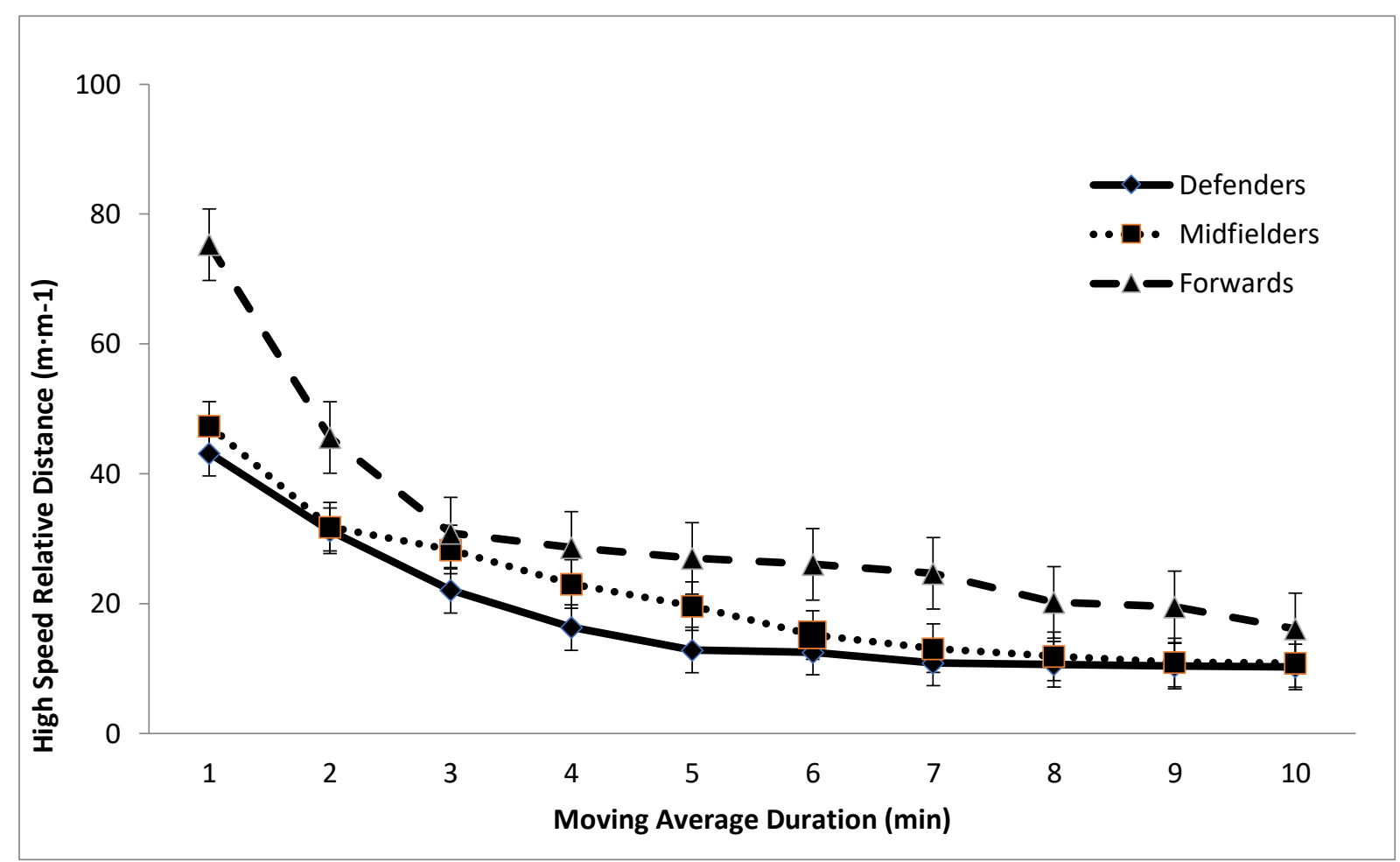

Fig 1. Maximum high-speed relative distances $(\mathrm{m} \cdot \mathrm{min}-1)$ of elite field hockey players by position for each moving average duration (mean). 


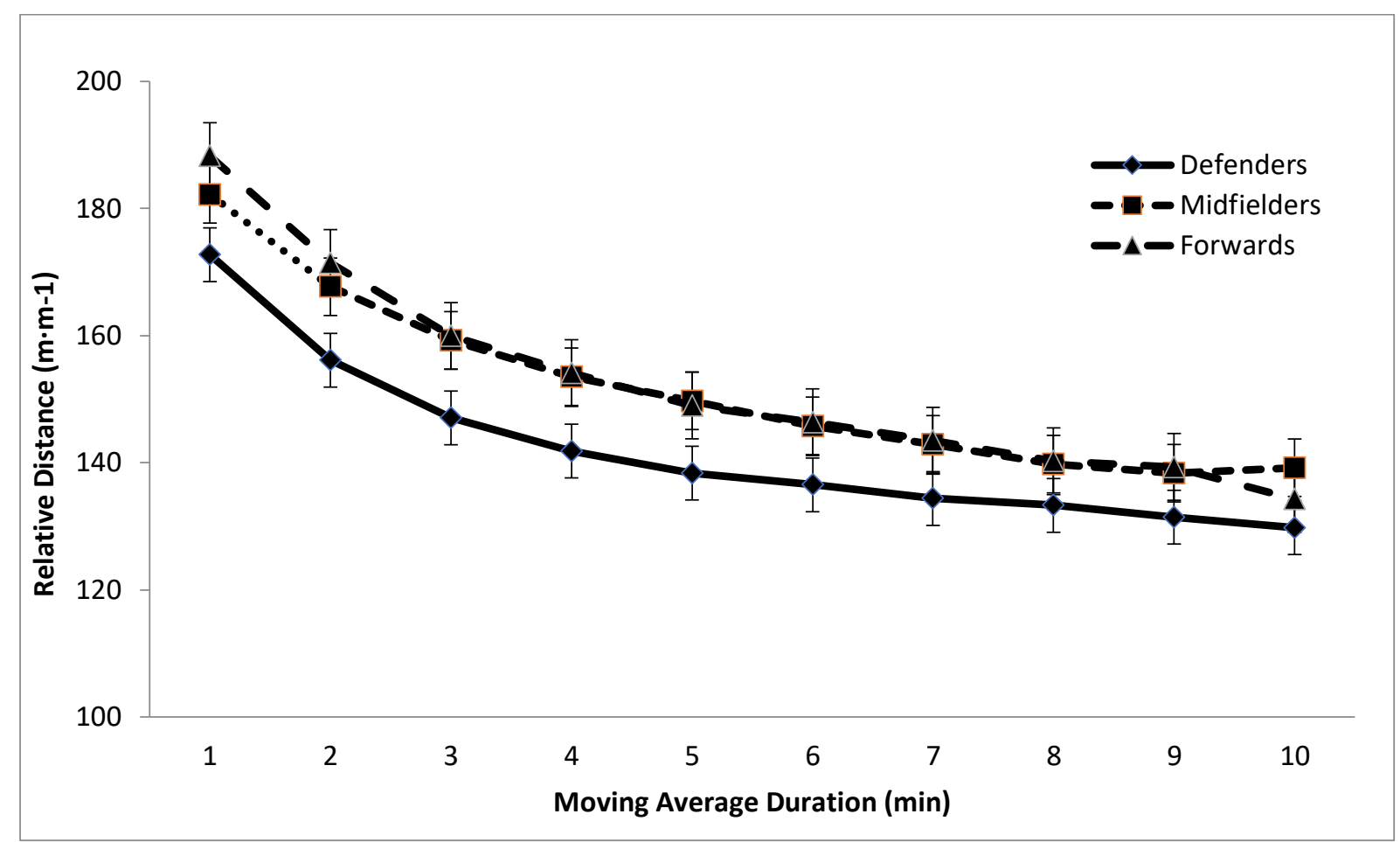

Fig 2. Maximum relative distances $(\mathrm{m} \cdot \mathrm{min}-1)$ of elite field hockey players by position for each moving average duration (mean). 


\begin{tabular}{|c|c|c|c|c|c|c|c|c|c|}
\hline & 1 & 2 & 3 & 4 & 5 & 6 & 7 & 8 & 9 \\
\hline \multirow[t]{2}{*}{2} & $15.99 \pm 1.94^{*}$ & & & & & & & & \\
\hline & Small $\uparrow$ & & & & & & & & \\
\hline \multirow[t]{2}{*}{3} & $25.65 \pm 2.06^{*}$ & $9.66 \pm 1.76^{*}$ & & & & & & & \\
\hline & Moderate $\uparrow$ & Small $\uparrow$ & & & & & & & \\
\hline \multirow[t]{2}{*}{4} & $31.25 \pm 2.16^{*}$ & $15.26 \pm 1.80^{*}$ & $5.59 \pm 1.67^{*}$ & & & & & & \\
\hline & Moderate $\uparrow$ & Moderate $\uparrow$ & Small $\uparrow$ & & & & & & \\
\hline \multirow[t]{2}{*}{5} & $35.37 \pm 2.23^{*}$ & $19.37 \pm 1.83^{*}$ & $9.71 \pm 1.66^{*}$ & $4.12 \pm 1.59 *$ & & & & & \\
\hline & Large $\uparrow$ & Moderate $\uparrow$ & Small $\uparrow$ & Small $\uparrow$ & & & & & \\
\hline \multirow[t]{2}{*}{6} & $38.18 \pm 2.29 *$ & $22.19 \pm 1.86^{*}$ & $12.52 \pm 1.66^{*}$ & $6.93 \pm 1.58^{*}$ & $2.81 \pm 1.51^{*}$ & & & & \\
\hline & Large $\uparrow$ & Moderate $\uparrow$ & Moderate $\uparrow$ & Small $\uparrow$ & Small $\uparrow$ & & & & \\
\hline \multirow[t]{2}{*}{7} & $40.84 \pm 2.34 *$ & $24.84 \pm 1.89 *$ & $15.18 \pm 1.67^{*}$ & $9.59 \pm 1.58^{*}$ & $5.47 \pm 1.48^{*}$ & $2.66 \pm 1.43^{*}$ & & & \\
\hline & Large $\uparrow$ & Large $\uparrow$ & Moderate $\uparrow$ & Small $\uparrow$ & Small $\uparrow$ & Small $\uparrow$ & & & \\
\hline \multirow[t]{2}{*}{8} & $43.31 \pm 2.40 *$ & $27.32 \pm 1.94^{*}$ & $17.66 \pm 1.70 *$ & $12.06 \pm 1.59 *$ & $7.95 \pm 1.49 *$ & $5.13 \pm 1.42 *$ & $2.48 \pm 1.35^{*}$ & & \\
\hline & Large $\uparrow$ & Large $\uparrow$ & Moderate $\uparrow$ & Moderate $\uparrow$ & Small $\uparrow$ & Small $\uparrow$ & Small $\uparrow$ & & \\
\hline \multirow[t]{2}{*}{9} & $44.70 \pm 2.43^{*}$ & $28.71 \pm 1.98 *$ & $19.04 \pm 1.74^{*}$ & $13.45 \pm 1.63^{*}$ & $9.33 \pm 1.51^{*}$ & $6.52 \pm 1.44^{*}$ & $3.86 \pm 1.36^{*}$ & $1.38 \pm 1.31^{*}$ & \\
\hline & Large $\uparrow$ & Large $\uparrow$ & Moderate $\uparrow$ & Moderate $\uparrow$ & Small $\uparrow$ & Small $\uparrow$ & Small $\uparrow$ & Small $\uparrow$ & \\
\hline \multirow[t]{2}{*}{10} & $46.65 \pm 2.42 *$ & $30.66 \pm 1.99 *$ & $21.00 \pm 1.76^{*}$ & $15.41 \pm 1.64 *$ & $11.29 \pm 1.52 *$ & $8.48 \pm 1.44^{*}$ & $5.82 \pm 1.35^{*}$ & $3.34 \pm 1.29 *$ & $1.96 \pm 1.27^{*}$ \\
\hline & Large $\uparrow$ & Large $\uparrow$ & Large $\uparrow$ & Moderate $\uparrow$ & Moderate $\uparrow$ & Small $\uparrow$ & Small $\uparrow$ & Small $\uparrow$ & Small $\uparrow$ \\
\hline
\end{tabular}

Table 2. Comparisons between maximum relative distances $(\mathrm{m} \cdot \mathrm{min}-1)$ for rolling averages of different durations

$*$ = significant difference between rolling average lengths $(p<0.05)$. Data are presented as mean $\pm 90 \% \mathrm{Cl}$, effect size and direction of effect. 


\section{DISCUSSION}

The present study was the first to investigate and determine the peak duration- and position-specific running intensities during field hockey competition, with velocity-based methods using a rolling average method of different durations. This study was able to describe the most intense period of a competitive game comparing different durations and positional groups, which can be used for prescription of training. ${ }^{6}$ Whilst positionspecific running demands of field hockey had been previously established, it had been limited to using averages across either an entire match ${ }^{3}$ or across each half 4, 5, 6, 18 . Recently, Delaney et al., (2015) utilised a rolling average method across 10 different durations to determine the peak running demands of rugby league competition, and compare these figures with previously reported absolute match data. The authors observed that rugby league match-play involves periods of play more intense than previously reported. ${ }^{24}$ This study shows that field hockey players spend $47 \pm 6$ min on the pitch, which is in agreement of previous research reporting a mean playing time of $44 \pm 7 \mathrm{~min}^{5}$ and 48 $\pm 4 \mathrm{~min}^{3}$. White and MacFarlane (2013) found significant differences in distance covered when comparing full game data to time spent on pitch data. This suggests the increase in recovery periods, allowing greater engagement with high-intensity running, also likening to fluctuate throughout the match, with some periods being more intense than others. ${ }^{3}$

The primary finding of this study was that the match-play running demands of field hockey players are significantly more intense than previously reported. A recent study by McGuiness et al., (2017) reported that on average, players covered a total distance of $126 \pm 23 \mathrm{~m} \cdot \mathrm{min}^{-1}$ regardless of playing position. A similar trend was observed in Division I College Field Hockey, reporting a total distance of $105 \pm 11$ $\mathrm{m} \cdot \mathrm{min}^{-1} .^{4}$ These observations are lower than the data shown in the present study, with players shown to cover a maximal relative distance of $181 \pm 24 \mathrm{~m} \cdot \mathrm{min}^{-1}$. With the relative distance data in the present study being greater than previously reported, it suggests that field hockey match-play involves periods of play which are considerable more intense than previously reported. The underestimation of demanding phases of play in field hockey is due to the highest activity period not matching pre-established time windows (0-5 $\mathrm{min}, 5-10 \mathrm{~min}$ etc). ${ }^{29}$ The trend observed in the present study was similar to that observed in rugby league players ${ }^{8}$, where the maximum running demands of competition significantly decreased as the duration of the moving average increased. The maximum intensity shown in this study could be used as a reference to program high intensity interval training matching the requirements of competitive matches. With the amount of time spent on the pitch per substitution averaging around 10 minutes, it highlights the importance of replicating the most demanding phases of play in training drills in time of equal duration. ${ }^{30}$

The majority of previous studies agree in suggesting that field hockey players spend most of a competitive match engaged in low-intensity exercise interspersed with short periods of high intensity, however a divide in results $\left(55 \%{ }^{3}, 95 \%{ }^{6}, 92 \%{ }^{7}\right)$ suggests different characterisations of low to moderate intensity exercise. 3 Nevertheless, this suggests that the 
utilization of high-speed running will vary throughout, with different positions engaging in intense situations at different stages throughout a match. Evidence suggests that situational variables are important factors for team sport performance, with match status, location and quality of opposition influencing the amount of high-speed running completed. ${ }^{26}$ The results of the current study suggest that regardless of position, maximal high-speed relative distance values $\left(55 \pm 18 \mathrm{~m} \cdot \mathrm{min}^{-1}\right)$ are much greater than previously reported. Vescovi and Frayne (2015) reported a high-speed relative distance of $10 \mathrm{~m} \cdot \mathrm{min}^{-1}$, seriously underestimating the running demands which could be of reason which leads to previous studies suggesting that field hockey training is not intense enough to reflect the physiological demands of match play. $^{2}$ Similarly, Macutkiewicz and Sunderland (2011) reported the average high-speed relative distance covered by players to be $\left(17.8 \pm 67 \mathrm{~m} \cdot \mathrm{min}^{-1}\right)$, however the differences in outputs are reflective of the absolute data collected, with the present study alternatively focussing on the most intense period of play. The differences in running intensities may be of practical use for the prescription and monitoring of field hockey training as the running requirements presented are maximum values attained reflecting the most intense block of running performed. Therefore, conditioning programs should be focussing on the most intense figures rather than the absolute data previously established.

A secondary aim of the study was to develop a position-specific velocity profile of field hockey competition. Differences in running demands were observed between positions, with significant differences observed across positional groups. Results show that forwards and midfielders covered a significantly $(p<0.05)$ greater maximal relative distance than defenders (F: $188 \pm 25 \mathrm{~m} \cdot \mathrm{min}^{-1} ; \mathrm{M}: 182 \pm 21 \mathrm{~m} \cdot \mathrm{min}^{-1}$; $\left.\mathrm{D}: 173 \pm 24 \mathrm{~m} \cdot \mathrm{min}^{-1}\right)$. There were no significant differences between the forwards and midfielders for maximal relative distances covered. Although there are differences between positions, the differences in relative distance covered may not be substantial enough to alter prescriptions of different intensities and positions. However, the differences between positions regarding high-speed running are substantial enough to alter the prescription of intensities. Figure 1 shows a significant difference in maximal highspeed relative distance covered between forwards and the other positions. Forwards covered a significantly greater maximal high-speed distance than midfielders and defenders (F: $75 \pm 14$ $\mathrm{m} \cdot \mathrm{min}^{-1} ; \mathrm{M}: 47 \pm 11 \mathrm{~m} \cdot \mathrm{min}^{-1} ; \mathrm{D} 43 \pm 9$ $\left.\mathrm{m} \cdot \mathrm{min}^{-1}\right)$, agreeing with recent reports suggesting that forwards spend a greater percentage of time performing highintensity exercise such as fast running and sprinting. ${ }^{3}$ It had been previously suggested that defenders cover more total distance than other positions due to more time spent on the pitch, however forwards and midfielders displaying greater high intensity running demands due to the nature of their positions. ${ }^{5}$ Similarly, Vescovi and Frayne (2015) reported that defenders and midfielders covered a greater distance $(\mathrm{m})$ over an entire match, however forwards engaged in the highest work rate $\left(\mathrm{m} \cdot \mathrm{min}^{-1}\right)$. This is likely a result of less playing time due to position-specific and tactical requirements due to the physiological demands of high-intensity running completed by forwards. The present study's results indicate that 
forwards are continuously seeking scoring opportunities which require high-speed movements in the opposition half, as well as off the ball movements to either seek space or apply pressure on the opposition's defence. The results of this study are supported by Vescovi and Frayne (2015) highlighting that forwards had greater proportions of maximal high-speed running than midfielders (10\%) and defenders (8.4\%). Recovery time between repeated sprints influences the completion of repeated high-intensity bouts. Due to a rule change in unlimited amount of substitutions, a lower playing time for forwards allows the ability to perform high-speed movements more frequently than other positions. ${ }^{27}$ Coaches use this information to allow differences in recovery time in order to have meaningful effects on performance, as well as in training to have impact on the tolerance to repeated high-intensity bouts. ${ }^{28}$

To conclude, the present study provides an insight into duration-specific and positionspecific running intensities and demands during field hockey competition in order to assist coaches in providing intensity specific training drills relative to peak match performance profiles. Results show that forwards and midfielders cover significantly more maximal relative distance than defenders, nevertheless highlighting that the match-play running demands within this study are significantly more intense than previously reported for all positions. When high-speed running was considered, forwards covered a significantly greater distance in maximal high-speed running compared to midfielders and defenders. This is in agreement with previous research suggesting that forwards engage in greater high-intensity exercise such as fast running and sprinting. ${ }^{3}$ The trend observed through the rolling average method showed that maximum running demands of competition significantly decreased as the duration of the moving average increased, showing the importance of replicating the most demanding phases of play in training drills in time of equal duration. A limitation of the present study is that due to only one team being analysis, it may be argued that the data presented may be reflective on the individual rather than actual playing position. As only one team was analysed, playing positions can be interchanged throughout a competitive match and throughout a season due to tactical reasons as well as match scenario's such as score line and opposition. As this was seen as a potential limitation approaching the study, the author attended each game and made notes of certain positional changes throughout the match, in order to make it as positionspecific as possible rather than an individualised approach. Due to squad sizes, the amount of players in certain positions such (e.g. defenders) were limited. The work rate required by forwards and midfielders requires a greater amount of substitutions whereas defenders are usually required to play the majority of the match. Overall, the present study provides maximal data on the running profiles of elite field hockey players. From these findings, it is suggested that coaches use the data obtained to implement position specific drills to replicate the demands of field hockey competition, especially regarding high-speed running. In order to reduce the likelihood of fatigue in players, it may be suggested that coaches replicate the most demanding phases of play in time of equal duration. 


\section{References}

1. Jennings DH, Cormack SJ, Coutts AJ, Aughey RJ. International field hockey players perform more high-speed running than national-level counterparts. The Journal of Strength \& Conditioning Research. 2012 Apr 1;26(4):947-52.

2. Gabbett TJ. GPS analysis of elite women's field hockey training and competition. The Journal of Strength \& Conditioning Research. 2010 May 1;24(5):1321-4.

3. Macutkiewicz D, Sunderland C. The use of GPS to evaluate activity profiles of elite women hockey players during match-play. Journal of Sports Sciences. 2011 Jun 1;29(9):967-73.

4. Vescovi JD, Frayne DH. Motion characteristics of division I college field hockey: Female Athletes in Motion (FAiM) study. International Journal of Sports Physiology and Performance. 2015 May 1;10(4):476-81.

5. McGuinness A, Malone S, Petrakos G, Collins K. The Physical and Physiological Demands of Elite International Female Field Hockey Players During Competitive Match-Play. Journal of strength and conditioning research. $2017 \mathrm{Jul} 24$.

6. MacLeod H, Morris J, Nevill A, Sunderland C. The validity of a non-differential global positioning system for assessing player movement patterns in field hockey. Journal of sports sciences. 2009 Jan 1;27(2):121-8.

7. Spencer M, Lawrence S, Rechichi C, Bishop D, Dawson B, Goodman C. Time-motion analysis of elite field hockey, with special reference to repeated-sprint activity. Journal of sports sciences. 2004 Sep 1;22(9):843-50.

8. Delaney JA, Scott TJ, Thornton HR, Bennett KJ, Gay D, Duthie GM, Dascombe BJ. Establishing duration-specific running intensities from match-play analysis in rugby league. International Journal of Sports Physiology \& Performance. 2015 Sep 1;10(6).

9. Delaney JA, Thornton HR, Rowell AE, Dascombe BJ, Aughey RJ, Duthie GM. Modelling the decrement in running intensity within professional soccer players. Science and Medicine in Football. $2018 \mathrm{Apr}$ 3;2(2):86-92.
10. Coutts AJ, Duffield R. Validity and reliability of GPS devices for measuring movement demands of team sports. Journal of science and Medicine in Sport. 2010 Jan 1;13(1):1335.

11. Scott MT, Scott TJ, Kelly VG. The validity and reliability of global positioning systems in team sport: a brief review. The Journal of Strength \& Conditioning Research. 2016 May 1;30(5):1470-90.

12. Aughey RJ. Applications of GPS technologies to field sports. International journal of sports physiology and performance. 2011 Sep 1;6(3):295-310.

13. White AD, MacFarlane N. Time-on-pitch or full-game GPS analysis procedures for elite field hockey?. International journal of sports physiology and performance. 2013 Sep 1;8(5):549-55.

14. Lacome M, Simpson BM, Cholley Y, Lambert $P$, Buchheit M. Small-Sided Games in Elite Soccer: Does One Size Fit All?. International journal of sports physiology and performance. 2018 May 1;13(5):568-76.

15. Lythe J, Kilding AE. Physical demands and physiological responses during elite field hockey. International Journal of Sports Medicine. 2011 Jul;32(07):523-8.

16. Furlan N, Waldron M, Shorter K, Gabbett TJ, Mitchell J, Fitzgerald E, Osborne MA, Gray AJ. Running-intensity fluctuations in elite rugby sevens performance. International journal of sports physiology and performance. 2015 Sep;10(6):802-7.

17. Delaney JA, Duthie GM, Thornton HR, Scott TJ, Gay D, Dascombe BJ. Acceleration-based running intensities of professional rugby league match play. International journal of sports physiology and performance. 2016 Sep 1;11(6):802-9.

18. Varley MC, Elias GP, Aughey RJ. Current match-analysis techniques' underestimation of intense periods of high-velocity running. International Journal of Sports Physiology and Performance. 2012 Jun 1;7(2):183-5.

19. Bradley PS, Noakes TD. Match running performance fluctuations in elite soccer: indicative of fatigue, pacing or situational influences?. Journal of sports sciences. 2013 Nov 1;31(15):1627-38.

20. Mohr M, Krustrup P, Bangsbo J. Match performance of high-standard soccer players 
with special reference to development of fatigue. Journal of sports sciences. $2003 \mathrm{Jan}$ 1;21(7):519-28.

21. Akenhead R, Hayes PR, Thompson KG, French D. Diminutions of acceleration and deceleration output during professional football match play. Journal of Science and Medicine in Sport. 2013 Nov 1;16(6):556-61.

22. Bradley PS, Di Mascio M, Peart D, Olsen $P$, Sheldon B. High-intensity activity profiles of elite soccer players at different performance levels. The journal of strength \& conditioning research. 2010 Sep 1;24(9):2343-51.

23. Bradley PS, Sheldon W, Wooster B, Olsen P, Boanas $P$, Krustrup P. High-intensity running in English FA Premier League soccer matches. Journal of sports sciences. 2009 Jan 1;27(2):159-68.

24. Gabbett TJ. Influence of playing standard on the physical demands of professional rugby league. Journal of Sports Sciences. 2013 Jun 1;31(10):1125-38.

25. Lythe J. The physical demands of elite men's field hockey and the effects of differing substitution methods on the physical and technical outputs of strikers during match play (Doctoral dissertation, Auckland University of Technology).

26. Lago C, Casais L, Dominguez E, Sampaio J. The effects of situational variables on distance covered at various speeds in elite soccer. European Journal of Sport Science. 2010 Mar 1;10(2):103-9.

27. Johnston T, Sproule J, McMorris T, Maile A. Time-motion analysis and heart rate response during elite male field hockey competition versus training. Journal of Human Movement Studies. 2004 Jan 1;46(3):189-203.

28. Billaut F, Basset FA. Effect of different recovery patterns on repeated-sprint ability and neuromuscular responses. Journal of sports sciences. 2007 Jun 1;25(8):905-13.

29. Sagarra ML, Campos-Vázquez MÁ. Differences of intermittency characterization during competitive matches: average values vs periods of maximum intensity.

30. Di Prampero PE, Fusi S, Sepulcri L, Morin JB, Belli A, Antonutto G. Sprint running: a new energetic approach. Journal of experimental Biology. 2005 Jul 15;208(14):2809-16. 